PATIENT SAFETY
A.P. Carlson
A.M. Brown
E. Zager
K. Uchino
M.P. Marks
C. Robertson

G.P. Sinson

A. Marmarou

H. Yonas

\title{
Xenon-Enhanced Cerebral Blood Flow at 28\% Xenon Provides Uniquely Safe Access to Quantitative, Clinically Useful Cerebral Blood Flow Information: A Multicenter Study
}

\begin{abstract}
BACKGROUND AND PURPOSE: Xe-CT measures CBF and can be used to make clinical treatment decisions. Availability has been limited, in part due to safety concerns. Due to improvements in CT technology, the concentration of inhaled xenon gas has been decreased from $32 \%$ to $28 \%$. To our knowledge, no data exist regarding the safety profile of this concentration. We sought to better determine the safety profile of this lower concentration through a multicenter evaluation of adverse events reported by all centers currently performing xenon/CT studies in the US
\end{abstract}

MATERIALS AND METHODS: Patients were prospectively recruited at 7 centers to obtain safety and efficacy information. All studies were performed to answer a clinical question. All centers used the same xenon delivery system. CT imaging was used during a 4.3-minute inhalation of $28 \%$ xenon gas. Vital signs were monitored on all patients throughout each procedure. Occurrence and severity of adverse events were recorded by the principal investigator at each site.

RESULTS: At 7 centers, 2003 studies were performed, 1486 (74.2\%) in nonventilated patients. The most common indications were occlusive vascular disease and ischemic stroke; $93 \%$ of studies were considered clinically useful. Thirty-nine studies (1.9\%) caused respiratory suppression of $>20$ seconds, all of which resolved spontaneously. Shorter respiratory pauses occurred in 119 (5.9\%), and hyperventilation, in 34 (1.7\%). There were 53 additional adverse events (2.9\%), 7 of which were classified as severe. No adverse event resulted in any persistent neurologic change or other sequelae.

CONCLUSIONS: Xe-CT CBF can be performed safely, with a very low risk of adverse events and, to date, no risk of permanent morbidity or sequelae. On the basis of the importance of the clinical information gained, $\mathrm{Xe}-\mathrm{CT}$ should be made widely available.

ABBREVIATIONS: $\mathrm{CBF}=$ cerebral blood flow; $E D=$ emergency department; FDA $=$ US Food and Drug Administration; ICP = intracranial pressure; IND = investigational new drug; $\mathrm{pCO}_{2}=$ carbon dioxide partial pressure; PRS = prolonged respiratory suppression; TIA = transient ischemic attack; $\mathrm{Xe}-\mathrm{CT}=$ xenon-enhanced $\mathrm{CT}$
A lthough previous single-institution experience has shown the safety of xenon, ${ }^{1}$ there remains concern in the literature and from the FDA related to potential respiratory side effects. Tens of thousands of Xe-CT studies have been performed worldwide in hundreds of centers, yielding accurate, detailed, and clinically useful information to guide patient

Received June 23, 2010; accepted after revision November 28.

From the Department of Neurosurgery (A.P.C., A.M.B., H.Y.), University of New Mexico, Albuquerque, New Mexico.; Department of Neurosurgery (E.Z.) University of Pennsylvania, Philadelphia, Pennsylvania; Department of Neurology (K.U.), University of Pittsburgh, Pittsburgh, Pennsylvania; Department of Neuroradiology (M.P.M.), Stanford University, Palo Alto, California; Department of Neurosurgery (C.R.), Baylor College of Medicine, Houston, Texas; Department of Neurosurgery (G.P.S.), Medical College of Wisconsin, Milwaukee, Wisconsin; and Division of Neurosurgery (A.M.), Medical College of Virginia, Richmond, Virginia.

Some institutions were supported in part by the National Institutes of Health for work contained herein.

Paper previously presented at: XIV World Congress of the World Federation of Neurosurgical Societies, August 30-September 4, 2009; Boston, Massachusetts.

Please address correspondence to Howard Yonas, MD, Department of Neurosurgery, University of New Mexico, MSC10-5615, 1 University of New Mexico, Albuquerque, NM 87131-0001; e-mail: hyonas@salud.unm.edu

Indicates open access to non-subscribers at www.ajnr.org

Indicates article with supplemental on-line table.

DOI 10.3174/ajnr.A2522 management. ${ }^{2,3}$ Despite an extensive clinical experience and no reported episode of any lasting sequelae related to $4.3 \mathrm{~min}-$ utes of $32 \%$ xenon inhalation, the literature continues to show concern for negative side effects due to xenon inhalation. These concerns are based on the respiratory suppression seen at very high anesthetic doses of xenon $(60 \%)$ and the incidence of minor self-limited pauses and other sensorial side effects seen at the lower diagnostic doses (32\%). Latchaw et $\mathrm{al}^{1}$ and Mathis et $\mathrm{al}^{4}$ previously reported the rates of adverse events at $32 \%$ xenon with respiratory pauses of $>10$ seconds occurring in $3.6 \%$. Other adverse events included headache $(0.4 \%)$, seizures $(0.2 \%)$, nausea and vomiting $(0.2 \%)$, and change in neurologic status $(0.1 \%) .{ }^{1}$ No report of persistent harm due to a Xe-CT CBF studies exists in the literature, to our knowledge, including the experience in Japan where the study has been in continuous wide use at $>400$ centers for the past decade.

Because side effects have remained of concern, the required concentration of inhaled xenon has been reduced to $28 \%$. This has been possible due to improvements in CT technology, which maintain the high signal intensity-to-noise ratio required for obtaining quantitative data. This reduction in concentration should further decrease any associated side effects.

$\mathrm{Xe}-\mathrm{CT}$ is one of only a few modalities capable of providing quantitative $\mathrm{CBF}$ data with high spatial resolution. These data 
should have an important role to play in the management of patients with complex neurologic problems. Xe-CT has been used in occlusive vascular disease, ${ }^{4-6}$ ischemic and hemorrhagic stroke, ${ }^{7,8}$ subarachnoid hemorrhage, ${ }^{9-11}$ other cerebrovascular disease, ${ }^{12,13}$ trauma, ${ }^{14-16}$ hydrocephalus, ${ }^{17}$ epilepsy,${ }^{18}$ tumor, ${ }^{19}$ psychiatric disease, ${ }^{20}$ and various pulmonary ${ }^{21}$ and hepatic ${ }^{22}$ applications. Due to the fast washout of xenon (around 3 minutes, with a physiologic half-life of approximately 30 seconds), serial Xe-CT CBF studies can be repeated in close temporal proximity. This is useful, for example, when testing for cerebrovascular reserve with acetazolamide or when assessing the utility of blood pressure augmentation in vasospasm. This technique is a powerful tool for determining hemodynamic insufficiency by identifying patients who have no vasodilatory reserve and, therefore, are at high risk of stroke. ${ }^{23}$ Worldwide, Xe-CT is becoming accepted as the standard for quantitative CBF measurement, ${ }^{24,25}$ so a detailed understanding of the safety profile of the technique is necessary. This is the first study, to our knowledge, to evaluate the sideeffect profile of $28 \%$ xenon in a wide spectrum of clinical disorders.

\section{Materials and Methods}

Xenon/CT CBF studies were performed as part of an IND protocol collecting safety and utility information. Participating centers included the University of New Mexico, University of Pennsylvania, University of Pittsburgh, Stanford University, Baylor College of Medicine, Medical College of Virginia, and the Medical College of Wisconsin. All studies were performed at the discretion of the treating physician to answer a clinical question relevant to providing the care of each patient (ie, for immediate treatment and patient-management decisions). Inclusion criteria and results of the study with regard to effectiveness in answering the clinical question were recorded.

The general procedure was standardized across institutions. Informed consent was obtained from the patient or legally authorized representative per the procedure defined by the institutional review board of each center. The study was performed either at the bedside (where a portable CT scanner was available) or at a fixed CT scanner. The bedside scanning potentially decreased patient transportationrelated morbidity and has been shown to consistently produce diagnostic-quality images. ${ }^{26}$ The CereTom (NeuroLogica, Danvers, Massachusetts) was the most commonly used dedicated head CT unit. It was an 8-section scanner, with a limiting spatial resolution of 7 line pairs per centimeter and radiation doses similar to those of a fixed scanner. ${ }^{26}$ Higher spatial resolution (as can be obtained with a fixed scanner with more detectors) was not required for this study. Adding more parallel detectors allowed more levels to be simultaneously studied but at higher radiation doses.

Patient selection was determined by the clinical judgment of the treating team, and indications were variable, but typically were related to cerebrovascular disease. When localizing scans had been performed, and several ${ }^{4-8}$ relevant CT sections were chosen, the patient was attached to the xenon machine, either with a mask or through the ventilator circuit. Standard vital signs were recorded before, throughout, and following each study; and when specialized monitors were in place such as intracranial pressure or cerebral oxygenation, these results were also recorded. The end-tidal xenon and end-tidal $\mathrm{CO}_{2}$ were continuously recorded by the xenon delivery system. Following the baseline scans, the patient began $28 \%$ xenon inhalation with the oxygen varying from $25 \%$ to $60 \%$, depending on the oxygenation needs of the patient. Most studies were performed with $28 \%$ xenon and $40 \%$ oxygen. Radiation dosing for a typical Xe-CT with the portable scanner at the University of New Mexico has been measured to be a $400-$ $\mathrm{mSv}$ average dose to the brain. This is equivalent to $40-\mathrm{mSv}$ effective dose equivalent to the whole body (personal communication; Philip H. Heintz, Diplomate of the American Board of Radiology). This is similar to published estimates of $39 \mathrm{mSv} .^{27}$

Calculation of CBF depends on a modification of the KetySchmidt technique for blood flow measurement. Rather than using the washout of a tracer, the equation is modified to measure the washin, a more reliable CBF estimate. To solve the integral equation, one must know the arterial input function as well as the brain arrival of the agent. Xenon is a rapidly diffusible gas, so the end-tidal xenon concentration is a very good approximation of the arterial concentra$\operatorname{tion}^{28}$ and avoids the need for either continuous arterial sampling or a single sample with an inferred concentration curve. The brain arrival is measured by the time-dependent brain xenon concentration as well as the brain-blood partition coefficient $(\lambda)$ by measuring the sequential change in Hounsfield units on each CT section. With these variables, the Kety-Schmidt equation is solved for each pixel of CT level chosen, yielding a quantitative CBF measure, which can then be grouped into regions of interest. ${ }^{2}$ The method has been extensively validated by using destructive methods (microspheres, ${ }^{29}$ iodoantipyrine $\left.^{24}\right)$ and in vivo methods $\left({ }^{133} \mathrm{xenon},{ }^{25}\right.$ positron-emission tomography, ${ }^{30}$ thermal dilution probes ${ }^{31}$ ) for measuring CBF.

After the 4.3-minute study, the xenon was turned off, and the end-tidal xenon was followed for a minimum of 1 minute to assess the washout time of xenon. The mask or ventilator circuit was then removed, and the patient returned to prestudy status. If desired, a second study could be performed as early as $5-10$ minutes after the first one to test the efficacy of an intervention. Integration of data, evaluation of quality of data, and the generation of flow data, which are operator-independent, required $<60$ seconds. The operator had the option of manually deleting poor-quality images to improve the level of confidence of the study. This analysis was all performed by using a software package developed for this application.

All adverse events were recorded by the investigator at each institution. Clinically significant adverse events were defined as follows: PRS is apnea $>20$ seconds; minimal respiratory suppression is a pause of 10-19 seconds; hyperventilation is a respiratory rate of $>25$ per minute; and an adverse event is any other reaction such as nausea, vomiting, anxiety, paresthesias, or loss of consciousness. The severity and outcome were determined at each institution. The investigator was also responsible for recording the clinical utility as to whether the study confirmed, clarified, or altered diagnosis or treatment.

\section{Results}

Between April 2002 and May 2009, 2003 Xe-CT studies were performed at 7 academic medical centers of varying sizes (Table 1). The most common indications for the studies included occlusive vascular disease $(n=705,35 \%)$, ischemic stroke $(n=537,27 \%)$, traumatic brain injury $(n=323,16 \%)$, and vasospasm after subarachnoid hemorrhage $(n=271,14 \%)$. Multiple indications were sometimes listed. Five hundred seventeen $(25.8 \%)$ patients were mechanically ventilated, and 211 $(40.7 \%)$ of these were chemically paralyzed. Most patients ( $n=1486,74.2 \%)$ were awake and not intubated. The studies were overall useful to patient care, with most altering, clarifying, or confirming diagnosis or treatment $(n=1862,93 \%)$; $141(7 \%)$ studies were of limited clinical value (Table 2). 


\begin{tabular}{ll}
\hline Table 1: Overall characteristics & \multicolumn{1}{c}{ No. } \\
\hline Characteristics & 2003 \\
\hline Total studies & \\
Location & $139(6.9 \%)$ \\
University of New Mexico & $170(8.5 \%)$ \\
University of Pennsylvania & $649(32 \%)$ \\
University of Pittsburgh & $710(35 \%)$ \\
Stanford & $236(12 \%)$ \\
Baylor College of Medicine & $82(4 \%)$ \\
Medical College of Wisconsin & $17(0.8 \%)$ \\
Medical College of Virginia & $517(25.8 \%)$ \\
Mechanically ventilated & $211(40.7 \%$ of ventilated) \\
Chemically paralyzed & $1486(74.2 \%)$ \\
Nonventilated & \\
Indication (some studies listed several) & $705(35 \%)$ \\
Occlusive vascular disease & $537(27 \%)$ \\
Ischemic stroke & $323(16 \%)$ \\
Traumatic brain injury & $271(14 \%)$ \\
Vasospasm after subarachnoid hemorrhage & $96(4.8 \%)$ \\
Hemorrhagic stroke & $62(3.1 \%)$ \\
Skull base aneurysm or tumor & $5(0.2 \%)$ \\
Epilepsy & $3(0.15 \%)$ \\
Brain tumor & $2(0.1 \%)$ \\
Dementia & $1(0.05 \%)$ \\
Hydrocephalus & $6(0.3 \%)$ \\
Other & \\
\hline
\end{tabular}

\begin{tabular}{lc}
\hline \multicolumn{2}{l}{ Table 2: Results of study } \\
\hline Results & No. $^{{ }^{a}}$ \\
\hline Confirmed diagnosis & $983(49 \%)$ \\
Clarified diagnosis & $779(39 \%)$ \\
Altered diagnosis & $61(3 \%)$ \\
Confirmed treatment & $861(43 \%)$ \\
Altered treatment & $258(13 \%)$ \\
Limited value & $141(7 \%)$ \\
\hline
\end{tabular}

a Studies may be classified in several categories.

Table 3 summarizes all adverse events. Respiratory events were the most commonly reported problems. Thirty-nine patients (1.9\%) had PRS and 119 (5.9\%) had minimal respiratory suppression, while $34(1.7 \%)$ had hyperventilation. All respiratory events except 1 accidental ventilator disconnect occurred in awake nonintubated patients. None of these events resulted in any consequence to any patient. The PRS events lasted between 20 seconds and 1 minute, with most in the range of 20-30 seconds. These events were generally associated with a decrease in end-tidal $\mathrm{pCO}_{2}$ and resolved as the $\mathrm{pCO}_{2}$ increased. This was generally thought to be due to the normal decreased respiratory drive in a low $\mathrm{pCO}_{2}$ and high $\mathrm{O}_{2}$ environment, and all cases resolved spontaneously in response to normalized $\mathrm{pCO}_{2}$ or cessation of the study.

Other adverse events occurred in 53 patients (2.6\%). Thirty-five of these were classified as mild and included a variety of events (Table 3). Ten patients had moderately severe events, including impaired consciousness, tremors, hypertension, dyspnea, taste disturbance, and numbness. All of these events resolved without further sequelae. Of all adverse events $(n=$ $53)$ and PRS events $(n=39), 12$ studies $(13 \%)$ required early termination.

Seven events $(0.35 \%)$ were classified as severe (On-line Table 1). The first was a patient with a severe traumatic brain injury. While in the CT scanner, the patient became brady-

\begin{tabular}{lr}
\hline Table 3: Adverse events & \\
\hline Event & No. \\
\hline Prolonged respiratory suppression (>20 seconds) & $39(1.9 \%)$ \\
Minimal respiratory suppression (10-19 seconds) & $119(5.9 \%)$ \\
Hyperventilation (>25/min) & $34(1.7 \%)$ \\
Other & $53(2.6 \%)$ \\
Nausea/vomiting & $11(0.5 \%)$ \\
Anxiety/funny feeling & $7(0.3 \%)$ \\
Agitation & $6(0.3 \%)$ \\
Hypertension & $5(0.2 \%)$ \\
Numbness/tingling & $4(0.2 \%)$ \\
Blurry vision & $4(0.2 \%)$ \\
Decreased/impaired level of consciousness & $4(0.2 \%)$ \\
Limb jerking/tremor & $2(0.1 \%)$ \\
Seizure & $2(0.1 \%)$ \\
Taste disturbance & $2(0.1 \%)$ \\
TIA-like symptoms & $2(0.1 \%)$ \\
Bradycardia to asystole & $1(0.05 \%)$ \\
Dyspnea & $1(0.05 \%)$ \\
Desaturation to 80 seconds & $1(0.05 \%)$ \\
Chest tightness & $1(0.05 \%)$ \\
Respiratory insufficiency & $1(0.05 \%)$ \\
Could not tolerate mask & $1(0.05 \%)$ \\
Headache & $1(0.05 \%)$ \\
Claustrophobia & $1(0.05 \%)$ \\
Severity & \\
Mild & $35(1.7 \%)$ \\
Moderate & $10(0.5 \%)$ \\
Severe & $7(0.3 \%)$ \\
Event required termination of study & 12 \\
\hline
\end{tabular}

cardic 5 minutes into the examination and progressed to asystole, requiring administration of epinephrine and atropine. Although the possibility that this event could have been related to the xenon study cannot be excluded, it was thought more likely to be due to the patient's severe underlying disease. The patient's neurologic status was unchanged after the incident.

During 2 of the studies, patients developed severe hypertension. One was in a patient with Takayasu arteritis with an ischemic stroke and baseline hypertension, and the other was in a patient with occlusive vascular disease and baseline hypertension. The first study was discontinued, and the blood pressure returned to baseline. The second study was completed with careful monitoring of the patient, and the patient's blood pressure returned to baseline after the study. Neither patient had any further events or complications. Two patients also developed seizures. The first patient was undergoing balloon test occlusion for a skull base aneurysm and had 1 seizure after balloon inflation and before xenon administration and a second seizure after xenon administration. The patient returned to baseline after the study, and the event was attributed to the study. The second was in a patient with occlusive disease, hemodynamic compromise, and TIAs, who had a seizure at the start of acetazolamide administration for a second xenon study. The examination was not completed, the patient was evaluated in the ED, and no other inciting factor or resultant problem was identified. The seizure was thought to be due possibly either to xenon, acetazolamide, or the patient's underlying cerebrovascular disease.

The next severe event was a patient with occlusive vascular disease and a history of stroke and myocardial infarct who developed anxiety and vomiting 4 minutes into the examina- 
tion. It was initially thought that the patient may have aspirated during the event, but the patient recovered without any sequelae or signs of aspiration. The anxiety may have been related to the xenon. The final severe event was in a patient who developed respiratory insufficiency after a second xenon study with acetazolamide challenge and required intubation. The patient was extubated in the ED with no sequelae. The event was thought likely to be an allergic reaction to the acetazolamide. There were no deaths or permanent morbidity or sequelae in the series.

\section{Discussion}

\section{Historical Considerations}

Xenon is an ideal contrast agent for the measurement of CBF because it is a very lipid soluble naturally occurring gas. Stable xenon is a noble gas, naturally occurring in the atmosphere with a concentration of $0.05 \%$. It is not only very lipid soluble but also radiopaque due to its molecular weight near iodine. This observation led to the novel CT-based assessment of flow based on the Kety-Schmidt equation, with the determination of CBF for each voxel reported by Gur et al. ${ }^{29}$ The use of stable xenon as a contrast agent intuitively seems likely to have a favorable safety profile. It is a noble (inert) gas so that there can be no allergic or lasting toxic reactions to xenon inhalation. Its high lipid solubility should be associated with a very rapid delivery and washout. To date, no lasting sequelae of xenon inhalation has been reported, to our knowledge.

Despite these observations, the noble gas xenon is not biologically inactive. High-dose xenon has been used as an inhalational anesthetic for half of a century. Recent studies that have shown not only efficacy as an anesthetic agent but also a very rapid onset and offset of action as well as no negative biologic effects will likely lead to more widespread use of the drug, despite the current high cost of manufacture. There are benefits in terms of analgesic, cardioprotective, ${ }^{32}$ and neuroprotective effects, presumably as an $N$-methyl-D-aspartate inhibitor. Xenon has been shown not to adversely affect respiratory or hemodynamic parameters and results in faster recovery from anesthesia compared with isoflurane. ${ }^{33}$ This record for safety in large clinical trials is based on inhalation for hours of $60 \%$ or higher concentrations of the xenon gas compared with the brief period of low-concentration xenon used in the current study for the measurement of CBF (28\%).

\section{Reported Safety Data in the Literature}

Safety data by using 32\% xenon inhalation for CBF have been reported in the literature by Latchaw et $\mathrm{al}^{1}$ as early as 1987. This series reported the safety profile in 1830 studies (the largest series to date). The most commonly observed adverse event was respiratory irregularity $(3.6 \%$ with a pause of $>10 \mathrm{sec}-$ onds). None of these respiratory problems were prolonged or serious. Other rare complications included headache $(0.4 \%)$, seizures $(0.2 \%)$, nausea and vomiting $(0.2 \%)$, and change in neurologic status $(0.1 \%) .{ }^{1}$ In the current series, the data were more rigorously prospectively collected under an FDA IND approval across multiple centers, some with high experience and some with less experience with the technology. These 2 factors likely explain why the reported rates of minor respiratory irregularity were lower in the previous series. The rate of severe events was lower than that in the previous series. In another series of 500 patients undergoing balloon test occlusion of the carotid artery with Xe-CT before carotid sacrifice, no significant complications could be attributed to the XeCT. ${ }^{4}$ In a 3 -year period by using approximately 200 bedside $\mathrm{Xe}-\mathrm{CT}$ studies in 75 patients, no adverse events related to the procedure were reported on the basis of both clinical observation and continuous vital sign monitoring, including ICP monitoring in patients with this available. ${ }^{34}$ This same group reported that $<5 \%$ of studies were interrupted for technical difficulties.

Chieregato et $\mathrm{al}^{35}$ reported experience with 180 xenon studies in 89 patients with severe traumatic brain injury and a Glasgow Coma Scale score of $<8$. No adverse events were reported, though the rates were not specifically addressed. ${ }^{35}$ Mori et $\mathrm{al}^{20}$ used Xe-CT to assess CBF in 118 patients with schizophrenia compared with healthy controls, demonstrating the feasibility of this technique even in a group of patients with potential behavioral challenges. No specific mention was made of technical limitations or complications in the series. Behrens et $\mathrm{al}^{36}$ (11 patients) used Xe-CT intraoperatively paired with stereotactic biopsy for gliomas. No adverse events were reported, and the minor difficulties of motion artifacts were eliminated with the rigid head fixation used for biopsy. One study showed some improved study quality with the use of a mild anxiolytic (oral hydroxyzine). Anxiety was the only adverse effect reported in this series of 120 patients. ${ }^{37}$ Worldwide, $\mathrm{Xe}-\mathrm{CT}$ has not only been more widely available but is also used in many non-CBF fields, further adding experience with it safety. Pulmonary and hepatic uses are most common, and reports of complications are minor and rare.

Overall, available data are consistent with regard to these effects. There does seem to be some incidence of respiratory pause with xenon administration, possibly related to hypocapnia and/or hyperoxygenation (in the potential case of baseline $\mathrm{CO}_{2}$ retention). A respiratory pause leads to return of more normal $\mathrm{CO}_{2}$ levels, which inevitably trigger spontaneous respiration via the normal physiologic mechanism. The pauses rarely cause even minor oxygen desaturation or other signs of adverse effects. Also, no effect continues after the cessation of the study. Likewise, there does seem to be some slight alteration in sensorium with patients; however, this immediately resolves after the study and is variably perceived by patients as pleasant or anxiety-provoking. The remainder of the anxiety effects are likely related simply to the study procedure (breathing through a mask in the CT scanner).

This is the largest study supporting the safety of the Xe-CT technique. Our results are consistent with much of the literature indicating that self-limited respiratory pauses and some mild alteration in sensorium ${ }^{38}$ are fairly common side effects, without permanent sequelae. ${ }^{39}$ It is true that a patient must remain still for an adequate study; however, this series showed a relatively low rate $(7 \%)$ of studies that were of limited clinical value. This is in contrast to previously reported high failure rates. ${ }^{40}$

As with any CT-based imaging technique, there is some risk of exposure to ionizing radiation, especially to the lens of the eye. This risk is minimized by positioning the gantry of the CT scanner so that the sections used in CBF data collection do not involve these structures. With this technique, no calcula- 
ble biologic effect on the lens or thyroid could be estimated. ${ }^{27}$ A whole-body equivalent dose has been estimated at $40 \mathrm{mSv}$ with $1 \mathrm{Xe}-\mathrm{CT}$ study. There is likely some small risk of oncogenesis with CT, though the exact dose and risk are controversial. One study estimated that the theoretic rate of fatal cancer formation was 1 per $12,500 \mathrm{Xe}-\mathrm{CT}$ studies. ${ }^{27}$ For this reason, it seems reasonable to focus the use of $\mathrm{Xe}-\mathrm{CT} \mathrm{CBF}$ studies in patients with serious disorders, in whom the benefit from having access to quantitative data and the ability to examine the response to physiologic challenges outweigh any theoretic radiation risk.

\section{Alternative Techniques}

A brief mention of other tomographic perfusion techniques is in order for perspective on the Xe-CT technique. CT and MR perfusion techniques rely on serial measurement of the arrival of a contrast bolus, similar to that in the Xe-CT technique. The difference is that the contrast agent is an intravascular rather than a diffusible tracer. These techniques are rapidly developing and will certainly aid in better physiologic understanding of the brain. Although CT and MR imaging contrast agents are widely used, generally safe, and approved by the FDA, they are not harmless. Iodinated contrast agents have improved in safety via newer formulations. Although some iodinated contrast concerns can be mitigated by patient selection and underlying disease, small risks of contrast nephropathy, ${ }^{41}$ allergic reaction, ${ }^{42}$ extravasation, ${ }^{43}$ and rarely death ${ }^{44}$ remain. With gadolinium-based agents, patient selection is once again important to decrease the risk of nephrogenic systemic fibrosis ${ }^{45}$ or other effects such as contrast nephropathy and extravasation. ${ }^{46}$ In critically ill patients, limitations in terms of vasoactive drips and other interventions such as ICP monitors also limit the ability to perform MR imaging. In contrast, no permanent death or disability has been reported with the use of xenon, though respiratory and other events as described in the article do occur.

\section{Conclusions}

Xenon/CT CBF is a safe technique for obtaining high-quality quantitative CBF data, which is useful for clinical decisionmaking in most cases. Risks and side effects are minimal and quickly reversible. Xenon gas has a proved safety record with no serious or life-threatening reported complications. Given the high degree of safety paired with reliable clinically relevant data, the use of this technique deserves to be expanded and made more widely available.

Disclosures: Howard Yonas, Ownership Interest: NeuroLogica, Details: Minor stock interest.

\section{References}

1. Latchaw RE, Yonas H, Pentheny SL, et al. Adverse reactions to xenon-enhanced CT cerebral blood flow determination. Radiology 1987;163:251-54

2. Pindzola RR, Yonas $H$. The xenon-enhanced computed tomography cerebral blood flow method. Neurosurgery 1998;43:1488-92

3. Vazquez Anon V, Aymard A, Gobin YP, et al. Balloon occlusion of the internal carotid artery in 40 cases of giant intracavernous aneurysm: technical aspects, cerebral monitoring, and results. Neuroradiology 1992;34:245-51

4. Mathis JM, Barr JD, Jungreis CA, et al. Temporary balloon test occlusion of the internal carotid artery: experience in $\mathbf{5 0 0}$ cases. AJNR Am J Neuroradiol 1995; 16:749-54

5. Carlson A, Yonas H. Monitoring carotid interventions with xenon CT. In: Gillard J, Graves M, Hatsukami T, et al, eds. Carotid Disease: The Role of Imaging in Diagnosis and Management. Cambridge, United Kingdom: Cambridge University Press; 2007:396-417
6. Webster MW, Makaroun MS, Steed DL, et al. Compromised cerebral blood flow reactivity is a predictor of stroke in patients with symptomatic carotid artery occlusive disease. J Vasc Surg 1995;21:338-44, discussion 344-45

7. Kitahara T, Yamashita T, Kashiwagi S, et al. Hemodynamics of hypertensive putaminal hemorrhage evaluated by xenon-enhanced computed tomography and acetazolamide test. Acta Neurol Scand Suppl 1996;166:139-43

8. Hughes RL, Yonas H, Gur D, et al. Cerebral blood flow determination within the first $\mathbf{8}$ hours of cerebral infarction using stable xenon-enhanced computed tomography. Stroke 1989;20:754-60

9. Carlson AP, Yonas H. Radiographic assessment of vasospasm after aneurysmal subarachnoid hemorrhage: the physiological perspective. Neurol Res 2009;31:593-604

10. Firlik KS, Firlik AD, Yonas H. Xenon-enhanced computed tomography in the management of cerebral vasospasm following aneurysmal subarachnoid hemorrhage. Keio J Med 2000;49(suppl 1):A148-50

11. Fukui MB, Johnson DW, Yonas $\mathrm{H}$, et al. Xe/CT cerebral blood flow evaluation of delayed symptomatic cerebral ischemia after subarachnoid hemorrhage. AJNR Am J Neuroradiol 1992;13:265-70

12. Tarr RW, Johnson DW, Rutigliano M, et al. Use of acetazolamide-challenge xenon CT in the assessment of cerebral blood flow dynamics in patients with arteriovenous malformations. AJNR Am J Neuroradiol 1990;11:441-48

13. Reigel MM, Hollier LH, Sundt TM Jr, et al. Cerebral hyperperfusion syndrome: a cause of neurologic dysfunction after carotid endarterectomy. J Vasc Surg $1987 ; 5: 628-34$

14. Hlatky R, Contant CF, Diaz-Marchan P, et al. Significance of a reduced cerebral blood flow during the first $\mathbf{1 2}$ hours after traumatic brain injury. Neurocrit Care 2004;1:69-83

15. Chieregato A, Noto A, Tanfani A, et al. Hyperemia beneath evacuated acute subdural hematoma is frequent and prolonged in patients with an unfavorable outcome: a Xe-computed tomographic study. Neurosurgery 2009;64:70517, discussion 717-18

16. Latchaw RE, Yonas H, Darby JM, et al. Xenon/CT cerebral blood flow determination following cranial trauma. Acta Radiol Suppl 1986;369:370-73

17. Maeder P, de Tribolet N. Xenon CT measurement of cerebral blood flow in hydrocephalus. Childs Nerv Syst 1995;11:388-91

18. Okuchi K, Nagata K, Otsuka H, et al. Regional cerebral blood flow after status epilepticus. Keio J Med 2000;49(suppl 1):A75-76

19. Van Roost D, Hartmann A, Quade G. Changes of cerebral blood flow following dexamethasone treatment in brain tumour patients: a Xe/CT study. Acta Neurochir (Wien) 2001;143:37-43, discussion 43-44

20. Mori K, Teramoto K, Nagao M, et al. Regional cerebral blood flow in schizophrenia using stable xenon-enhanced computed tomography. Neuropsychobiology 1999;39:117-24

21. Goo HW, Chae EJ, Seo JB, et al. Xenon ventilation CT using a dual-source dual-energy technique: dynamic ventilation abnormality in a child with bronchial atresia. Pediatr Radiol 2008;38:1113-16

22. Gur D, Good WF, Herbert DL, et al. Blood flow mapping in the human liver by the Xe-CT method. J Comput Assist Tomogr 1985;9:447-50

23. Yonas H, Smith HA, Durham SR, et al. Increased stroke risk predicted by compromised cerebral blood flow reactivity. J Neurosurg 1993;79:483-89

24. Wolfson SK Jr, Clark J, Greenberg JH, et al. Xenon-enhanced computed tomography compared with [14C] iodoantipyrine for normal and low cerebral blood flow states in baboons. Stroke May 1990;21:751-57

25. Matsuda M, Lee H, Kuribayashi K, et al. Comparative study of regional cerebral blood flow values measured by Xe CT and Xe SPECT. Acta Neurol Scand Suppl 1996;166:13-16

26. Rumboldt Z, Huda W, All JW. Review of portable CT with assessment of a dedicated head CT scanner. AJNR Am J Neuroradiol 2009;30:1630-36. Epub 2009 Aug 6

27. Seifert $\mathrm{H}$, Blass $\mathrm{G}$, Leetz $\mathrm{HK}$, et al. The radiation exposure of the patient from stable-xenon computed tomography. Br J Radiol 1995;68:301-05

28. Gur D, Good WF, Wolfson SK, Jr, et al. In vivo mapping of local cerebral blood flow by xenon-enhanced computed tomography. Science 1982;215:1267-68

29. Gur D, Yonas H, Jackson DL, et al. Simultaneous measurements of cerebral blood flow by the Xe-CT method and the microsphere method: a comparison. Invest Radiol 1985;20:672-77

30. Nariai T. Comparison of measurement between Xe/CT CBF and PET in cerebrovascular disease and brain tumor. Acta Neurol Scand Suppl 1996;166:10-12

31. Valadka AB, Hlatky R, Furuya Y, et al. Brain tissue PO2: correlation with cerebral blood flow. Acta Neurochir Suppl 2002;81:299-301

32. Preckel B, Weber NC, Sanders RD, et al. Molecular mechanisms transducing the anesthetic, analgesic, and organ-protective actions of xenon. Anesthesiology 2006;105:187-97

33. Rossaint R, Reyle-Hahn M, Schulte Am Esch J, et al. Multicenter randomized comparison of the efficacy and safety of xenon and isoflurane in patients undergoing elective surgery. Anesthesiology 2003;98:6-13

34. Sturnegk P, Mellergard $\mathrm{P}$, Yonas $\mathrm{H}$, et al. Potential use of quantitative bedside CBF monitoring (Xe-CT) for decision making in neurosurgical intensive care. Br J Neurosurg 2007;21:332-39

35. Chieregato A, Tanfani A, Compagnone C, et al. Global cerebral blood flow and 
CPP after severe head injury: a xenon-CT study. Intensive Care Med 2007;33:856-62

36. Behrens PF, Ostertag CB, Warnke PC. Regional cerebral blood flow in peritumoral brain edema during dexamethasone treatment: a xenon-enhanced computed tomographic study. Neurosurgery 1998;43:235-40, discussion $240-41$

37. Sesay M, Tanaka A, Dousset V, et al. Effects of premedication with oral hydroxyzine on patient motion during inhalation of $32 \%$ xenon for regional cerebral blood flow mapping. Keio J Med 2000;49(suppl 1):A41-44

38. Yonas H, Grundy B, Gur D, et al. Side effects of xenon inhalation. J Comput Assist Tomogr 1981;5:591-92

39. Yonas H, Pindzola RP, Johnson DW. Xenon/computed tomography cerebral blood flow and its use in clinical management. Neurosurg Clin $\mathrm{N} \mathrm{Am}$ 1996;7:605-16

40. Harrod CG, Bendok BR, Batjer HH. Prediction of cerebral vasospasm in patients presenting with aneurysmal subarachnoid hemorrhage: a review. $\mathrm{Neu}$ rosurgery 2005;56:633-54
41. Krol AL, Dzialowski I, Roy J, et al. Incidence of radiocontrast nephropathy in patients undergoing acute stroke computed tomography angiography. Stroke 2007;38:2364-66

42. Wang CL, Cohan RH, Ellis JH, et al. Frequency, outcome, and appropriateness of treatment of nonionic iodinated contrast media reactions. AJR Am J Roentgenol 2008;191:409-15

43. Schaverien MV, Evison D, McCulley SJ. Management of large volume CT contrast medium extravasation injury: technical refinement and literature review. J Plast Reconstr Aesthet Surg 2008;61:562-65, discussion 565

44. Ray P, Riou B, Beigelman C, et al. Case reports of death after nonionic iodine contrast medium injection during spiral computed tomography scan performed for suspected pulmonary embolism. Am J Emerg Med 2006;24:497-99

45. Shellock FG, Spinazzi A. MRI safety update 2008. Part 1. MRI contrast agents and nephrogenic systemic fibrosis. AJR Am J Roentgenol 2008;191:1129-39

46. Bellin MF, Van Der Molen AJ. Extracellular gadolinium-based contrast media: an overview. Eur I Radiol 2008;66:160-67 